\title{
Subgroup Analysis Makes GOLD2019's New Comprehensive Assessment More Convincing冈A Cross-Sectional Study in Hunan Province, China
}

\section{Zhongshang Dai}

Second Xiangya Hospital of Central South University

Yan Chen ( $\sim$ chenyan99727@csu.edu.cn )

Second Xiangya Hospital of Central South University

Huihui Zeng

Second Xiangya Hospital of Central South University

Yanan Cui

Second Xiangya Hospital of Central South University

\section{Research Article}

Keywords: analysis, comprehensive assessment, cross-sectional study, Hunan Province, severity, disease, patient, medical center

Posted Date: July 20th, 2021

DOI: https://doi.org/10.21203/rs.3.rs-712096/v1

License: (c) (i) This work is licensed under a Creative Commons Attribution 4.0 International License. Read Full License 


\section{Abstract}

Purpose: To estimate the severity of the disease in outpatients with chronic obstructive pulmonary disease (COPD) in Hunan Province, China and use the subgroup analysis to evaluate the reliability of Global Initiative for Chronic Obstructive Lung Disease (GOLD) 2019.

Methods: COPD outpatients from 21 medical centers in Hunan Province, China were stratified into groups $A-D$, and group $D$ patients were further stratified into subgroups $D_{1}-D_{3}$ according to the GOLD 2016 comprehensive assessment. Demography, clinical characteristics and medications were compared between groups.

Results: In 1,017 COPD outpatients, the distribution from group $A$ to $D$ and subgroup $D_{1}$ to $D_{3}$ was 41 (4.0\%), 249 (24.5\%), 17 (1.7\%), 710 (69.8\%) and 214 (30.2\%), 204 (28.7\%), 292 (41.1\%), according to GOLD 2016. The clinical characteristics of patients vary from group to group. Some patients in groups $C-D$ regrouped to groups $A-B$ were all $C_{1}$ and $D_{1}$ subgroups according to GOLD 2019. Comparing subgroup $D_{1}$ with group $B$, group $D$, subgroup $D_{2}$ and subgroup $D_{3}$, it was found that the demography, clinical characteristics and medications of subgroup $D_{1}$ were the closest to group $B$, according to GOLD 2016.

Conclusion: The disease severity of outpatients with COPD in Hunan Province was more common in group $B$ and $D$ and patients in groups A- D had different demography, clinical characteristics and medications, according to the GOLD 2016. Subgroup analysis can explain to a certain extent that GOLD2019's new comprehensive assessment is more reasonable and reliable than GOLD 2016.

\section{Introduction}

Chronic obstructive pulmonary disease (COPD) is a global public health challenge due to its high prevalence and related mortality. ${ }^{1}$ The most recent China Pulmonary Health (CPH) study reported the overall prevalence of spirometry-defined COPD was $8.6 \%$ among the general Chinese population aged 20 years or older and the estimated total number of individuals was 99.9 million. ${ }^{2}$ However, no Hunan Province data are available for severity of COPD outpatients.

The clinical management of COPD was mostly guided by the Global Initiative for Chronic Obstructive Lung Disease (GOLD) document. From GOLD 2011 to GOLD 2016, patients were stratified by ABCD assessment tool, which incorporated symptoms, spirometry measure, and frequency of exacerbations. Lange et al also performed analyses of the subgroups of the $C$ and $D$ categories, as patients can be stratified into these categories through different scenarios. ${ }^{3}$ Thus, categories $C$ and $D$ were subdivided into subgroups $C_{1}, C_{2}, C_{3}, D_{1}, D_{2}$, and $D_{3}$. They found groups $C$ and $D$ are heterogeneous, being composed of phenotypes with variable risk. In the 2019 update of GOLD document, a refinement of the ABCD assessment tool was proposed that separated spirometry measure from the $A B C D$ group. ${ }^{4}$ Because of the brand new assessment tool, Some patients in groups C-D were regrouped to groups A-B. However, 
we are not sure whether the refined $A B C D$ assessment scheme is more suitable for the COPD population grouping than the previous creterion.

In this study, we aimed to estimate the severity of the disease in outpatients with COPD in Hunan Province, China. Furthermore, we use the subgroup analysis to evaluate the reliability of Global Initiative for Chronic Obstructive Lung Disease (GOLD) 2019.

\section{Methods}

\section{Study participants}

The study was a cross-sectional observational survey in Hunan Province, China. From January 1, 2017 to December 31, 2017, Patients were recruited from COPD outpatient clinics from 21 tertiary hospitals in Hunan Province, China. The study was approved by the ethics review committee of the Second Xiangya Hospital, Central South University and the ethics registration number was: No. ChiCTR-POC-17010431. We got informed consent from all study participants.All methods were carried out in accordance with COPD guidelines and regulations. ${ }^{1}$

Patients who were recruited had a clear diagnosis of COPD according to the GOLD 2016, based on the persistent airflow limitation defined as post-bronchodilator FEV1/forced vital capacity (FVC) $<70 \%$. Furthermore, the patients were in a stable stage, that was, there was no acute exacerbation within one month and they were proficient in Chinese with no communication barriers. Patients suffering from other diseases that cause airflow limitation or decreased lung capacity were excluded.

\section{Study procedure}

The standardised questionnaire was distributed to each patient to record demographic characteristics and medical history data including name, gender, smoking history, the COPD assessment test (CAT) questionnaire, the modified Medical Research Council (mMRC) questionnaire, the clinical COPD (CCQ) questionnaire, spirometry results, exacerbation history, maintenance treatment modalities, and so on. We defined the history of occupational exposure as working in an environment with silicious dust, graphite dust, coal dust or asbestos during the past 6 months or longer. We defined the history of biofuel contact as heating woody type fuels or animal waste during the past 6 months or longer. We defined formersmokers as quitting smoking for more than 6 months. We defined the course of chronic bronchitis/emphysema as cough and sputum production for at least 3 months in each of two consecutive years. ${ }^{1}$ We divided the maintenance treatment modalities into the following 8 different programs of drugs: a short-acting bronchodilators (short-acting $\beta 2$ receptor agonists or short-acting anticholinergics, SABA/SAMA) , a long-acting bronchodilators (long-acting $\beta 2$ receptor agonists) or longacting anticholinergic, LABA/LAMA), a short-acting bronchodilators + a long-acting bronchodilator (SABA/SAMA+LABA/LAMA), a long-acting $\beta 2$ agonist + a long-acting anticholinergic (LABA+LAMA), a long-acting $\beta 2$ receptor agonist + an inhaled hormone combination (LABA + ICS), a short-acting bronchodilator + a long-acting $\beta 2$ receptor agonist $+a$ inhaled hormone (SABA/SAMA+LABA+ICS), a 
long-acting $\beta 2$ receptor agonist + a long-acting anticholinergic + an inhaled hormone (triple), a shortacting bronchodilator + a long-acting $\beta 2$ receptor agonist + a long-acting anticholinergic + an inhaled hormone (triple + SABA / SAMA) .

According to the GOLD 2016 comprehensive assessment, ${ }^{1}$ patients were stratified on the basis of symptoms with either CAT score $(<10$ or $\geq 10)$ or mMRC score $(0-1$ vs $\geq 2)$ resulting in two low-symptom categories ( $A$ and $C$ ) and two high-symptom categories ( $B$ and $D$ ). Exacerbation risk was assessed with either COPD exacerbation history ( 0 vs $\geq 1$ hospitalization) / ( $0-1$ vs $\geq 2$ ) or FEV $1 \%$ predicted $(<50 \%$ or $\geq 50 \%$ ), in the previous year resulting in two low-risk groups ( $A$ and $B$ ) and two high-risk groups( $C$ and D). When the exacerbation risk, as assessed by FEV $1 \%$ predicted value, or the exacerbation history were not identical, risk was defined by the method showing the greater risk. An exacerbation was defined as an acute event characterized by a worsening of the patient's respiratory symptoms that is beyond normal day-to-day variations and leads to a change in medication.

We also made analyses of the subgroups of the GOLD $C$ and $D$, as patients can be further stratified into these categories through different scenarios: ${ }^{1}$

$\mathrm{C}_{1}$ or $\mathrm{D}_{1}: \mathrm{FEV} 1<50 \%$ pred and fewer than two exacerbations (and $<1$ hospitalized exacerbation) in the previous year

$\mathrm{C}_{2}$ or $\mathrm{D}_{2}$ : FEV1 $\geq 50 \%$ pred and two or more exacerbations(or $\geq 1$ hospitalized exacerbation ) in the previous year

$\mathrm{C}_{3}$ or $\mathrm{D}_{3}$ : FEV1 $<50 \%$ pred and two or more exacerbations (or $\geq 1$ hospitalized exacerbation )in the previous year

\section{Statistical analysis}

The statistical analysis including statistical description and statistical inference were performed with software SPSS version 21.0. The age, body mass index (BMI), smoking index, the course of chronic bronchitis/emphysema, hospitalizations in the previous year, exacerbations in the previous year, FEV1\% predicted, FEV1/FVC, mMRC, and CAT were all measurement data without normal distribution, being expressed as the median (interquartile range). The gender, COPD family history, education level, occupational exposure history, biofuel contact history, smoking history and comorbidities were all counting data, being expressed as the number (composition ratio). For measurement data without normal distribution, the Kruskal-Wallis $H$ test was used to compare the $A, B, C$, and $D$ groups and the Wilcoxon test was used to compare each two groups. The chi-square test was used to compare the counting data between the groups. Forest plot using standardized mean difference of measurement data and odds ratio of counting data was used to compare subgroup $D_{1}$ with group $B$, group $D$, subgroup $D_{2}$ and subgroup $D_{3}$. 


\section{Results}

Patient distribution by GOLD2007, 2016 and 2019 comprehensive assessment methods between January 1, 2017, and December 31, 2017, 1296 outpatients were selected and invited to participate in the survey. 279 patients were excluded in which 28 patients were unable to read questionnaires, 132 patients had no spirometry results, 47 patients did not meet the COPD diagnostic criteria, and 72 patients had other active respiratory diseases, and 1017 patients were included in the final analysis (Figure 1). Of the 1017 patients recruited to this study, the distribution of comprehensive assessment groups according to GOLD 2007: Grade I was $8.0 \%(81 / 1,017)$, Grade II was $41.4 \%(421 / 1,017)$, Grade III was $38.0 \%(387 / 1,017)$, and Grade IV was $12.6 \%(128 / 1,017)$. Using the GOLD 2016 comprehensive assessment, group A was $4.0 \%$ $(41 / 1,017)$, B was $24.5 \%$ (249/1,017), C was $1.7 \%$ (17/1,017), and D was $69.8 \%(710 / 1,017)$. Using the GOLD 2019 comprehensive assessment, group A was 4.6\% (47/1,017), B was 45.5\% (463/1,017), C was $1.1 \%(11 / 1,017)$, and D was $48.8 \%(496 / 1,017)$ (Figure 2$)$. Therefore, the disease severity of outpatients with COPD in Hunan Province was more common in group B and D. Group D patients were further stratified into subgroups $D_{1}-D_{3}$ according to the GOLD 2016: $D_{1}$ was $30.2 \%(214 / 710), D_{2}$ was $28.7 \%$ (204/710), $D_{3}$ was $41.1 \%(292 / 710)$. Group C patients were unable to perform subgroup analysis and statistical analysis because of small sample sizes.

\section{Demography, clinical characteristics and medications of groups $A, B, C, D$}

In the groups $A, B, C$, and $D$, there was no significant difference in gender ratio, family history of COPD, history of exposure to biofuel, and smoking index. The proportion of high-education, FEV1/FVC, and FEV $1 \%$ pred in group $A$ was significantly higher than that in group $B, C$, and $D$, while the occupational exposure history, course of disease, and questionnaire scores were significantly lower than those in groups B, C, and D. The clinical characteristics of group B were more prominent. The proportion of current smoking patients, BMI index, and the proportion of coronary artery disease in group B were the highest, and the proportion of former-smokers was the lowest among the groups A, B, C, and D. Patients in group $C$ had no specific clinical features and the lowest proportion. The age, course of disease, exacerbations in the previous year, and questionnaire scores were significantly higher in the $D$ group than in the $A, B$, and $C$ groups, and FEV1/FVC, FEV1\% pred were significantly lower than those in the A, B, and C groups(Table 1).

Among the actual drug prescriptions received by patients in groups $A, B, C$, and $D$, LAMA is the most widely used, followed by triple therapy and LABA+ICS. Among the patients in group $A$, the patients receiving LAMA were the most, followed by the triple therapy. Patients in group $B$ also received the most treatment with LAMA, followed by LABA+ICS. The patients who received the most frequently prescribed therapy in groups $C$ and $D$ were treated with triple therapy, followed by LAMA(Figure 3,4$)$.

\section{Demography, clinical characteristics and medications of subgroups D}

In the subgroups $D_{1}, D_{2}$, and $D_{3}$, there was no significant difference in age, family history of COPD, history of exposure to biofuel, education level, CCQ score and occupational exposure history. The proportion of current smoking patients, BMI index, and the proportion of coronary artery disease in 
subgroup $D_{1}$ were the highest, whereas the proportion of former-smokers was the lowest among the subgroups $D_{1}, D_{2}$, and $D_{3}$. Besides, the exacerbations in the previous year was significantly lower in subgroup $D_{1}$ than in subgroups $D_{2}, D_{3}$. The proportion of female patients, never-smokers, FEV1/FVC, and FEV1\%pred in group $D_{2}$ was significantly higher than in subgroups $D_{1}, D_{3}$, and the smoking packyears was significantly lower than in subgroups $D_{1}, D_{3}$. The course of disease, $C A T$, and mMRC scores in group $D_{3}$ was higher than in subgroups $D_{1}, D_{2}$ (Table 2 ).

There is a large difference in the proportion of drug prescriptions among patients in the D subgroup. The drug treatment in the $\mathrm{D}_{1}$ group accounted for the largest proportion of LAMA alone, followed by LABA+ICS. The $D_{2}$ group had the highest number of patients with triple therapy, followed by LAMA alone. The $D_{3}$ group also had the highest number of patients with triple therapy, followed by LABA+ICS (Figure $5)$.

\section{Subgroup analysis confirmed that GOLD 2019 was more reliable}

According to the comprehensive assessment of the new GOLD2019 document, some patients in groups C-D were regrouped to groups $A-B$, especially 214 patients (30.2\%) in group $D$ were regrouped to group $B$. However, in this study, we found that the groups $C$ and $D$ regrouped to groups $A$ and $B$ were all subgroups $C_{1}$ and $D_{1}: 6$ patients (35.3\%) in subgroup $C_{1}$ were regrouped to group $A$, and 214 patients $(30.2 \%)$ in subgroup $D_{1}$ were regrouped to group $B$, which suggested that patients in the subgroups $C_{1}$ and $D_{1}$ may have the similar demographic and clinical characteristics as patients in groups $A$ and $B$ (Figure 6). By further comparing the four groups $B$, subgroup $D_{1}$, subgroup $D_{2}$ and subgroup $D_{3}$, it was found that the demography, clinical characteristics and medications of subgroup $D_{1}$ were the closest to group B, especially in the proportion of current smoking patients, BMI index, coronary artery disease, former-smokers, and maintenance medication. It's not hard to see that it was actually more appropriate to stratify subgroup $D_{1}$ into group $B$. So we came to the conclusion that GOLD2019's new comprehensive assessment is more reasonable and reliable than GOLD 2016. (Figure 7).

\section{Discussion}

To the best of our knowledge, this study is the first survey focusing on the distribution, clinical characteristics and medications of COPD patients recruited from the outpatient clinics in Hunan, China. At the same time, we are committed to use the subgroup analysis to evaluate the changes of definition in assessment of GOLD stratification 2019. In our study we found that the disease severity of outpatients with COPD in Hunan Province was more common in group B and $D$, especially in group $D$. This consequence was similar to that reported in the previous COPD case-cohort studies recruited from hospital clinics ${ }^{19,26,27}$, but was obviously different from the situation dominated by group A in COPD patients identified in the general population ${ }^{17,18,20}$. This result may be considered because patients who came to the hospital for medical help often had more serious symptoms and a longer course of disease, meanwhile most patients with early COPD were unaware of their condition and few had performed a 
previous pulmonary function test, let alone saw a doctor. So the distribution of patient categories in an area may be substantially different based on different populations and patient selection criteria. It also suggested that epidemiological studies were of great significance for the prevention and early detection of COPD using spirometry, which should be a public health priority in Hunan Province.

As we knew, the core change of refinement of the brand new ABCD assessment tool was the shift of removing spirometry measure and leaving the symptoms and frequency of exacerbations in the categorization, that brought about some patients from high-risk groups without an exacerbation history in the previous year shifting to the low-risk groups. As a result, when classified by the GOLD 2019 assessment, we found that in Cabrera and Lina Sun's national large-scale cross-sectional studies, they showed more than one third of high-risk groups were regrouped to low-risk groups and group A occupied the largest proportion of patients ${ }^{36,16}$. However, group D was still the biggest group in our study, although the proportion of patients in group B was significantly higher than before, according to the revised 2019 $A B C D$ classification. Neven Tudoric et al also presented the similar result that group $B$ and $D$ were the most prevalent groups in their POPE cohort ${ }^{28}$. This phenomenon was considered because the group $D$ in our province had a large base number, and group $\mathrm{C}$ was the smallest. Even if one third of patients were reclassified, the proportion of group composition was not changed, but the proportion of patients in group D had a significant downward trend compared with the group document of GOLD 2016.

In this study, patients in group A had a short course of disease, fewer occupational exposures, and the highest proportion of those with higher education. This is similar to the results of previous literature studies. A disease-related analysis from Poland divides the educational level into three levels according to the number of years of education. The level of education increases by one level, and the population has chronic obstructive pulmonary disease. The risk was reduced by about $35 \%$, while the patients in Group A were mainly concentrated at the high education level ${ }^{29}$. Eisner's findings show that patients with high education levels are more likely to have early detection of the disease, a stronger awareness of the diagnosis, a lower lung function than those with a lower education, and a lower risk of acute exacerbations in the future ${ }^{30}$. Academician Wang Chen also found that only about $10 \%$ of the respondents knew about the disease of "slow obstruction lung disease", and less than $3 \%$ of patients knew they had chronic obstructive pulmonary disease. It is pointed out that among the various factors related to chronic obstructive pulmonary disease, "low education level" is an important part that can not be ignored ${ }^{7}$. Patients in group A have high academic qualifications, and have a strong awareness of disease and early treatment; however, the level of personal education cannot be changed in a short time, but public health education can be strengthened through various channels to raise public awareness of chronic obstructive pulmonary disease, for example COPD has been renamed as "slow-resistance lung", so that the common people can know and remember; and strive to make the public recognize chronic obstructive lungs like cognitive hypertension and diabetes. The clinical characteristics of group B were more prominent. The proportion of current smoking patients in group B was the highest, the proportion of patients who quit smoking was the lowest, and the BMI index was the highest. Other studies have similar results. Agusti A and other studies also found that group B patients with C-reactive protein and other 
inflammatory indicators were significantly higher than other groups ${ }^{26}$, considering the group B patients with systemic symptoms may be related to systemic inflammatory response, but This study did not collect comparative analysis of inflammatory factor specimens; another Agusti $A$ also found that patients in groups $A, B, C$, and $D$ were followed up for three years and found that $35 \%$ of patients in group $B$ were converted to group $D$, while patients with group $A, C$, and D were grouped. There was no significant change before, suggesting that patients in group $B$ had large clinical variability and fluctuating conditions, and their symptoms and progress of lung function should be closely monitored. Therefore, for patients with group $B$, the decline in lung function is not obvious, but the symptoms are serious. The conditions of extrapulmonary diseases such as obesity, inflammatory reaction, cardiovascular disease, etc. should be highly valued and actively treated. The proportion of patients in group $C$ was the lowest, as was the case with previous clinical epidemiological studies ${ }^{20}$, suggesting that lung function is poor and respiratory symptoms are rare. This phenomenon may be related to the generally high drug reactivity in patients in group $\mathrm{C}$ and the improvement in symptoms after treatment. Therefore, the promotion and promotion of knowledge related to chronic obstructive pulmonary disease should be strengthened to improve patients' understanding of the disease and their awareness of treatment. The duration of chronic bronchitis/emphysema was significantly higher in group $D$ than in group $A, B$, and $C$. The predicted value of FEV1\% was significantly lower than that in group $A, B$, and $C$, and the age was higher than that in group A, B, and C; Risk, symptoms are related to the course of chronic bronchitis / emphysema, lung function, age. Several clinical studies abroad have also suggested that age is associated with progressive decline in lung function and frequent exacerbations ${ }^{31,32}$. Therefore, for patients with long course of disease, progressive decline in lung function may be difficult to avoid, and the incentive to actively prevent acute exacerbation is a key measure.

The clinical characteristics, acute exacerbation and drug treatment of the subgroup $D$ patients were significantly different. The D1 group had the highest proportion of current smoking patients, the lowest smoking cessation rate, the highest BMI index, and the proportion of drug prescription distribution, which were similar to those in group $B$, and the difference was not statistically significant $(p<0.01)$. According to GOLD2017, all D1 groups were assigned to group B, suggesting that the clinical characteristics of group D1 and group B are similar ${ }^{1}$, which also indirectly confirms that the new comprehensive evaluation method of GOLD2017 is more conducive to disease judgment and treatment guidance. The D2 group had the largest number of female patients, the highest number of never smokers, and the lowest smoking index, which is consistent with foreign studies. The results of Landis et al showed that gender differences in patients with chronic obstructive pulmonary disease are closely related to smoking, and current smokers are concentrated in male patients ${ }^{33}$. Kohansal et al found that the average annual decline in lung function in smoking patients was faster and the disease worsened significantly higher than nonsmokers ${ }^{34}$, and Burney et al also found that pulmonary dysfunction was closely related to smoking index 35. Academician Wang Chen pointed out that the prevalence of chronic obstructive pulmonary disease in smoking population was $13.7 \%$, which was more than $1 \%$ in non-smokers; the prevalence of chronic obstructive pulmonary disease in smoking population over 60 years old was over $40 \%$; the longer smoking time, the amount of smoking The greater the risk of chronic obstructive pulmonary disease, the 
higher the risk ${ }^{7}$. All of these suggest that smoking is associated with progressive impairment of lung function; and whether it is GOLD2011, GOLD2016, GOLD2017 documents, emphasis is placed on smoking cessation as the primary prevention and treatment of chronic obstructive pulmonary disease. Therefore, the promotion of tobacco control is the most important factor in the prevention and control of chronic obstructive pulmonary disease. The measures that have been proven to effectively delay the progressive decline of lung function have only been to quit smoking. Therefore, the World Health Organization's Framework Convention on Tobacco Control should be strictly implemented to strengthen tobacco control propaganda, highlighting the serious harm of smoking in various forms; popularizing clinical smoking cessation services, such as brief smoking cessation services, smoking cessation clinics and smoking cessation hotlines. Patients with D3 had the highest duration of chronic bronchitis/emphysema, but Lange et al did not investigate the duration of chronic bronchitis/emphysema 17; the other D3 patients were treated with triple therapy, while the D1 patients were treated with D3. The main use of a long-acting bronchodilator. Clinical studies such as Chen Yahong in China suggest that the key to re-classification into high-risk and low-risk groups is the use of inhaled corticosteroids ${ }^{36}$. Combined with the results of this study, patients in the D1 group were considered to have a low frequency of acute exacerbation, more stable symptom control, and more drug treatments with long-acting bronchodilator alone, but less hormones; therefore, the D1 group was completely reclassified to the high-risk group. Low risk group. The patients in the D3 group showed that the number of acute exacerbations increased and the lung function damage increased; therefore, the excessive use of inhaled glucocorticoids needs attention. The patients in group D were followed up. The acute exacerbation rate of patients in D2 and D3 group was significantly higher than that in D1 group. The condition of D1 group was stable for a long time, which is consistent with foreign studies. Han et al. found in the COPDgene study that there was a significant difference in the frequency of acute exacerbations in each of the $D$ groups, with the highest in the D3 group, followed by the D2 group and the D1 group ${ }^{20}$. Lange et al also found that the 1-and 3-year mortality rates of the D3 group were higher than those of the D1 and D2 groups ${ }^{17}$. All of these suggest the importance of acute exacerbation for the grouping of chronic obstructive pulmonary disease; and attaching importance to subgroups in patients with $C$ and $D$, it is more conducive to accurate and comprehensive assessment of the condition, thus contributing to the long-term management of patients with chronic obstructive pulmonary disease.

This study is a multi-center study that is prone to information bias and confounding bias; and does not analyze all relevant confounding factors and may interfere with the results. In addition, this study only targeted some patients with chronic obstructive pulmonary disease in Hunan Province, and could not fully reflect the severity of disease in patients with chronic obstructive pulmonary disease in Hunan Province. Patients in group D did not have a long follow-up period and may differ slightly from the final study. Therefore, in future research, patients in group $D$ should be followed up, and their acute exacerbations and prognosis should be analyzed and compared. Blood samples can be collected, and relevant indicators such as inflammatory factors can be analyzed and compared.

\section{Conclusion}


The disease severity of outpatients with COPD in Hunan Province was more common in group B and D and patients in groups A- D had different demography and clinical characteristics, according to the GOLD 2016. Subgroup analysis can explain to a certain extent that GOLD2019's new comprehensive assessment is more reasonable and reliable than GOLD 2016. The guiding value of the new disease assessment method for judging the prognosis of COPD patients needs further study. Subgroup analysis can explain to a certain extent that GOLD2019's new comprehensive assessment is more reasonable and reliable than GOLD 2016.The guiding value of the new disease assessment method for judging the prognosis of COPD patients needs further study.

\section{Declarations}

\section{Declaration of interest}

There are no conflicts of interest of all authors in this study.

\section{References}

[1] Vogelmeier CF, Criner GJ, Martinez FJ, et al. Global strategy for the diagnosis, management, and prevention of chronic obstructive lung disease 2017 report. GOLD executive summary. Am J Respir Crit Care Med. 2017;195(5):557-582.

[2] López-Campos JL, Tan W, Soriano JB. Global burden of COPD [ J ].Respirology, 2016,21 ( 1 ) : 14 -23 .

[3] Fang X,Wang X, Bai C.COPD in China:the burden and importance of proper management[J]. Chest, 2011, 139(4): 920-929.

[4]Zhong N, Wang C, Yao W, et al. Prevalence of chronic obstructive pulmonary disease in China冈a large

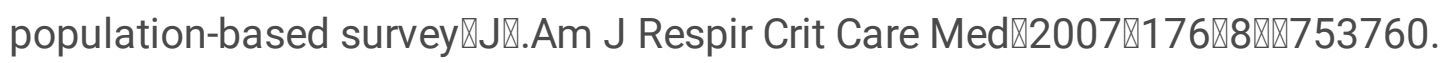

[6] GBD 2015 Disease and Injury Incidence and Prevalence Collaborators. Global, regional, and national incidence, prevalence, and years lived with disability for 310 diseases and injuries, 1990-2015: a systematic analysis for the Global Burden of Disease Study 2015. Lancet 2016; 388: 1545-602.

[7] Chen Wang, et al. Prevalence and risk factors of chronic obstructive pulmonary disease in China (the China Pulmonary Health [CPH] study): a national cross- sectional study [J]. Lancet, 2018, published online.

[8] Rabe KF, Hurd S, Anzueto A, et al. Global strategy for the diagnosis, management, and prevention of chronic obstructive pulmonary disease: GOLD executive summary. Am J Respir Crit Care Med. 2007;176(6): 532-555.

[9] Hurst JR, Vestbo J, Anzueto A, et al. Susceptibility to exacerbation in chronic obstructive pulmonary disease. N Engl J Med. 2010;363(12): 1128-1138. 
[10] Agusti A, Calverley P, Celli B, et al. Characterization of COPD heterogeneity in the ECLIPSE cohort. Respir Res. 2010;11:122.

[11] Han MK, Agusti A, Calverley PM, et al. Chronic obstructive pulmonary disease phenotypes. Am J Respir Crit Care Med. 2010;182(5): 598-604.

[12] Divo M, Cote C, de Torres JP, et al. Comorbidities and risk of mortality in patients with chronic obstructive pulmonary disease. Am J Respir Crit Care Med. 2012;186(2):155-161.

[13] Vestbo J, Hurd SS, Agustí AG, et al. Global strategy for the diagnosis, management, and prevention of chronic obstructive pulmonary disease: GOLD executive summary. Am J Respir Crit Care Med. 2013; 187(4):347-365.

[14] Soriano JB, Lamprecht B, Ramirez AS, et al. Mortality prediction in chronic obstructive pulmonary disease comparing the GOLD 2007 and 2011 staging systems: a pooled analysis of individual patient data. Lancet Respir Med. 2015;3(6):443-450.

[15] Goossens LM, Leimer I, Metzdorf N, Becker K, Rutten-van Molken MP. Does the 2013 GOLD classification improve the ability to predict lung function decline, exacerbations and mortality: a post-hoc analysis of the 4-year UPLIFT trial. BMC Pulm Med. 2014;14:163.

[16] Kim J, Yoon HI, Oh YM, et al. Lung function decline rates according to GOLD group in patients with chronic obstructive pulmonary disease. Int J Chron Obstruct Pulmon Dis. 2015;10:1819-1827.

[17] Lange P, Marott JL, Vestbo J, et al. Prediction of the clinical course of chronic obstructive pulmonary disease, using the new GOLD classification: a study of the general population [J]. Am J respir Crit Care Med, 2012, 186: 975-981.

[18]Johannessen A, Nilsen RM, Storeb $\emptyset$ M, et al.Comparison of 2011 and 2007 Global Initiative for Chronic Obstructive Lung Disease guidelines for predicting mortality and hospitalization [J]. Am J Respir Crit Care Med, 2013, 188:51-59.

[19] Soriano JB, Alfageme I, Almagro P, et al. Distribution and prognostic validity of the new Global Initiative for Chronic Obstructive Lung Disease grading classification [J]. Chest, 2013,143:694-702.

[20] Han MK囚Mullerova H囚Curran-Everett D囚et al『GOLD 2011 disease severity classification in COPDgene:

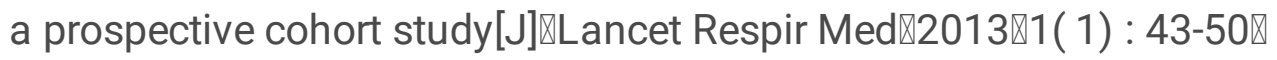

[22] Global strategy for the diagnosis, management and prevention of chronic obstructive pulmonary disease (updated 2016)[EB/OL]. (2016-01) [2016-04-05]. http://www.goldcopd.org.

[23] Halpin DM, Decramer M, Celli B, et al. Exacerbation frequency and course of COPD[J]. Int J Chron Obstruct Pulmon Dis. 2012;7:653-61. 
[24] Bestall JC, Paul EA, Garrod R, et al.Usefulness of the Medical Research Council (MRC) dyspnoea scale as a measure of disability in patients with chronic obstructive pulmonary disease[J]. Thorax 1999, 54(7):581-586.

[25] Jones PW, Harding G, Berry P, et al.Development and first validation of the COPD Assessment Test[J]. Eur Respir J 2009,34(3):648-654.

[26] Agusti A, Edwards LD, Celli B, et al. Characteristics, stability and outcomes of the 2011 GOLD COPD groups in the ECLIPSE cohort. Eur Respir J. 2013;42(3):636-646.

[27] Sahadevan A, Cusack R, O'Kelly B, Amoran O, Lane SJ. The Value of the combined assessment of COPD in accurate characterization of stable COPD. Ir Med J. 2016;109(1):338-340.

[28] Tudoric N, Koblizek V, Miravitlles M, et al. GOLD 2017 on the way to a phenotypic approach? Analysis from the phenotypes of COPD in central and eastern Europe (POPE) cohort. Eur Respir J. 2017;49(4): 1602518.

[29]Nizankowska-Mogilnicka E1, Mejza F, Buist AS, et al. Prevalence of COPD and tobacco smoking in Malopolska region--results from the BOLD study in Poland[J]. Pol Arch Med Wewn. 2007 Sep;117(9):40210.

[30]Eisner MD, Blanc PD, Omachi TA, et al. Socioeconomic status, race and COPD health outcomes[J]. J Epidemiol Community Health. 2011 Jan;65(1):26-34.

[31]Santibanez M, Garrastazu R, Ruiz-Nuñez M, et al. Predictors of hospitalized exacerbations and mortality in chronic obstructive pulmonary disease. PLoS One. 2016;11(6):e0158727.

[32] Gudmundsson G, Ulrik CS, Gislason T, et al. Long-term survival in patients hospitalized for chronic obstructive pulmonary disease: a prospective observational study in the Nordic countries. Int J Chron Obstruct Pulmon Dis. 2012;7:571-576.

[33]Landis SH, Muellerova H, Mannino DM, et al. Continuing to confront COPD international patient survey: methods, COPD prevalence, and disease burden in 2012-2013. Int J Chron Obstruct Pulmon Dis. 2014;9: 597-611.

[34] Kohansal R, Martinez-Camblor P, Agustí A, et al. The natural history of chronic airflow obstruction revisited: an analysis of the Framingham offspring cohort. Am J Respir Crit Care Med. 2009;180(1):3-10.

[35]Burney P, Jithoo A, Kato B, et al; Burden of Obstructive Lung Disease (BOLD) Study. Chronic obstructive pulmonary disease mortality and prevalence: the associations with smoking and poverty a BOLD analysis. Thorax. 2014;69(5):465-473.

[36] lina sun, Yahong Chen et al. Changes in definition lead to changes in the clinical characteristics across COPD categories according to GOLD 2017: a national crosssectional survey in China. International 
Journal of COPD 2017:12 3095-3102.

[37] Drivenes E, Ostrem A, Melbye H. Predictors of ICS/LABA prescribing in COPD patients: a study from general practice[J].BMC Fam Pract. 2014 Mar 5;15:42.

[38] Thomas M, Radwan A, Stonham C, et al. COPD exacerbation frequency, pharmacotherapy and resource use: an observational study in UK primary care[J]. COPD. 2014 Jun;11(3):300-9.

[39] Magnussen H, Disse B, Rodriguez-Roisin R, et al. Withdrawal of inhaled glucocorticoids and exacerbations of COPD[J]. N Engl J Med. 2014 Oct 2;371(14):1285-94.

\section{Tables}

Due to technical limitations, table 1-2 is only available as a download in the Supplemental Files section.

\section{Figures}


1296 outpatients were invited to participate in the survey

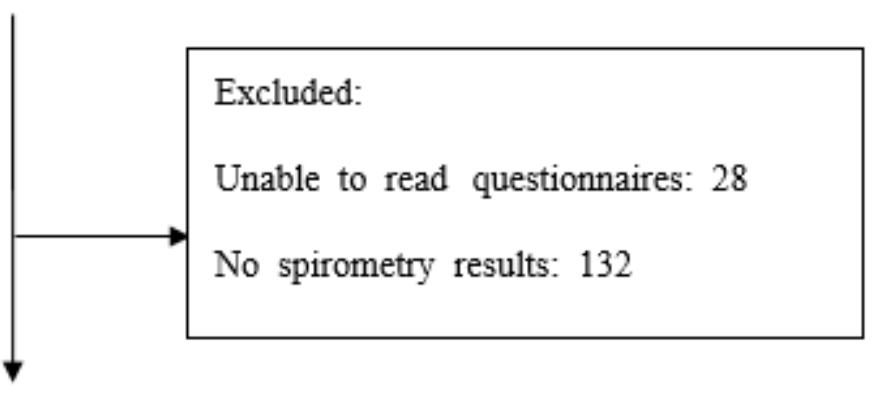

1017 final outpatients for analysis

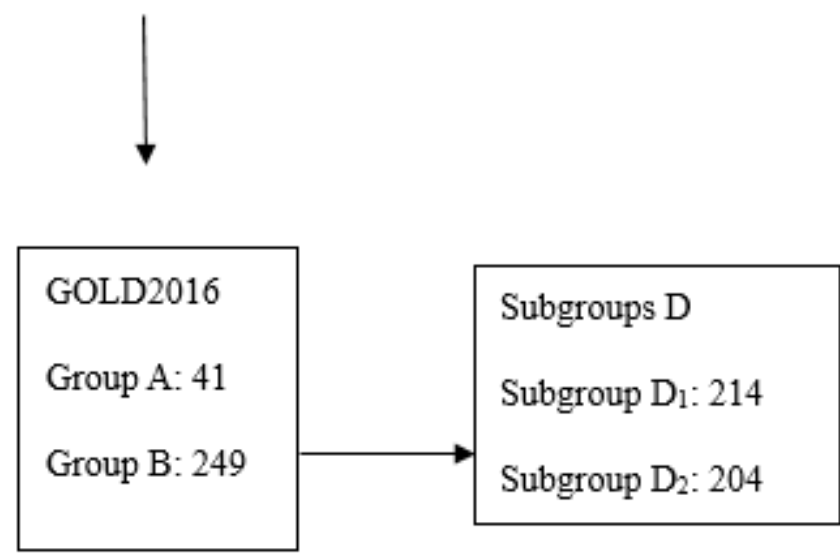

\section{Figure 1}

Study flow chart. 


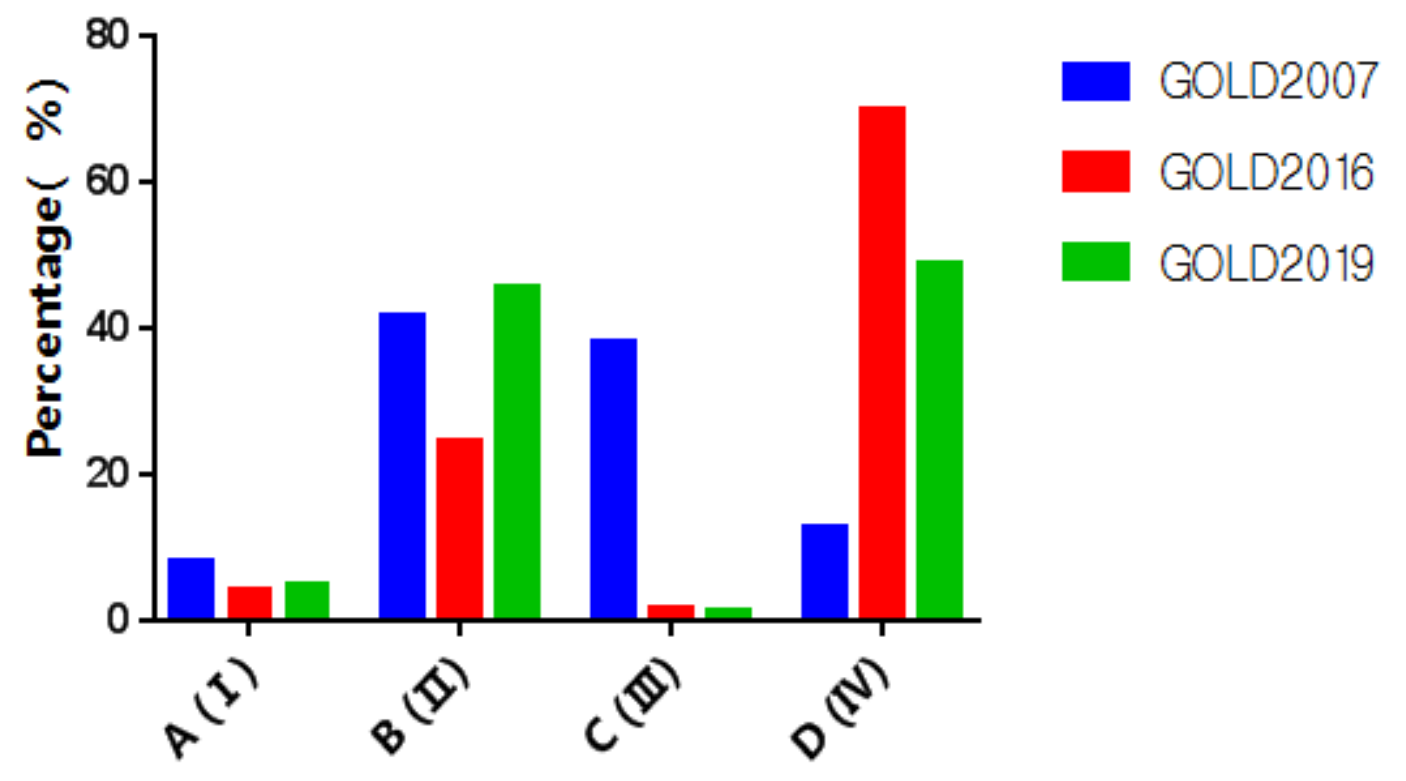

Figure 2

Distribution of groups A-D of the (GOLD 2007), (GOLD 2016) and (GOLD 2019) comprehensive assessment. Abbreviation: GOLD, global Initiative for Chronic Obstructive lung Disease. 


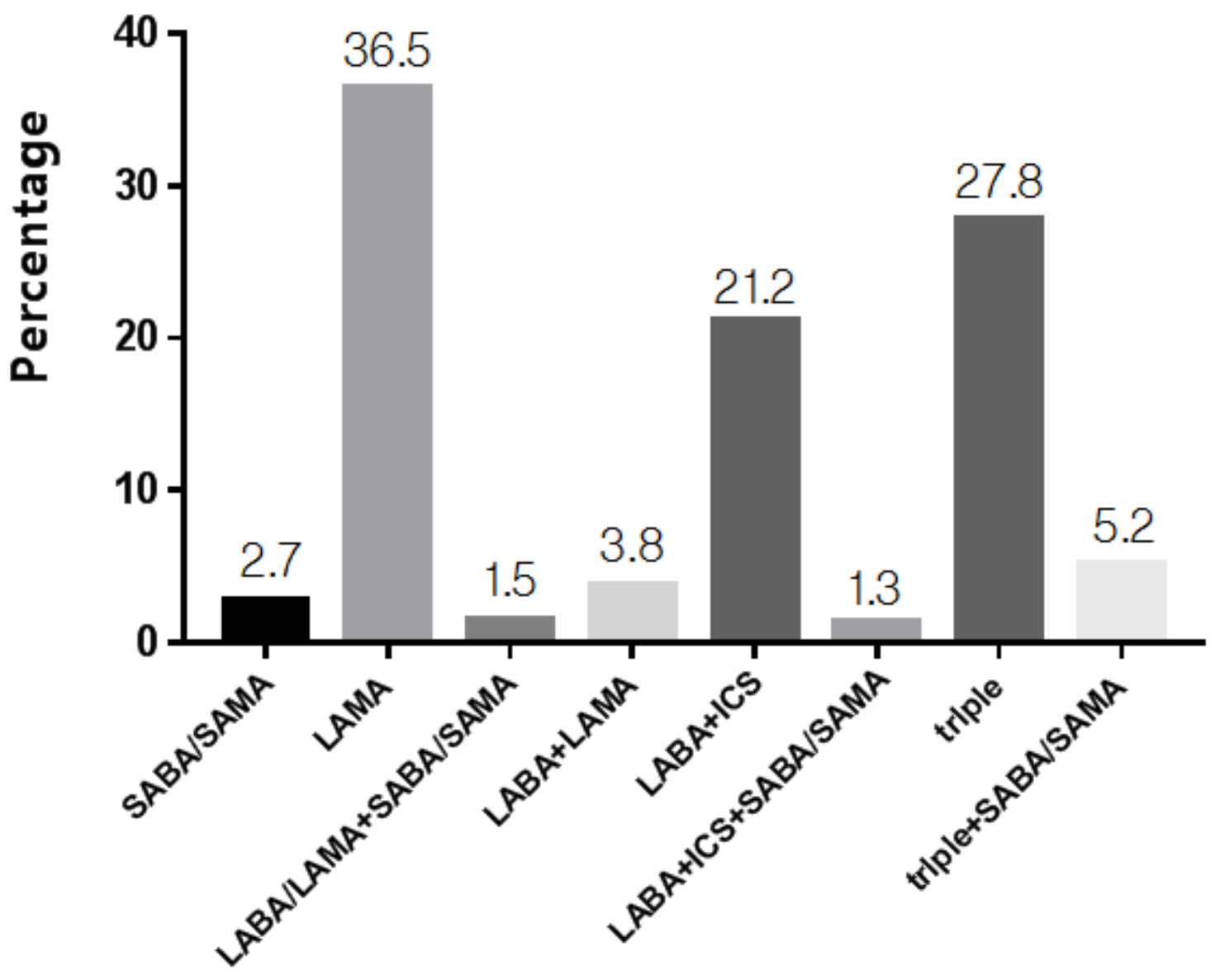

Figure 3

Distribution of medications in 1017 patients with COPD 

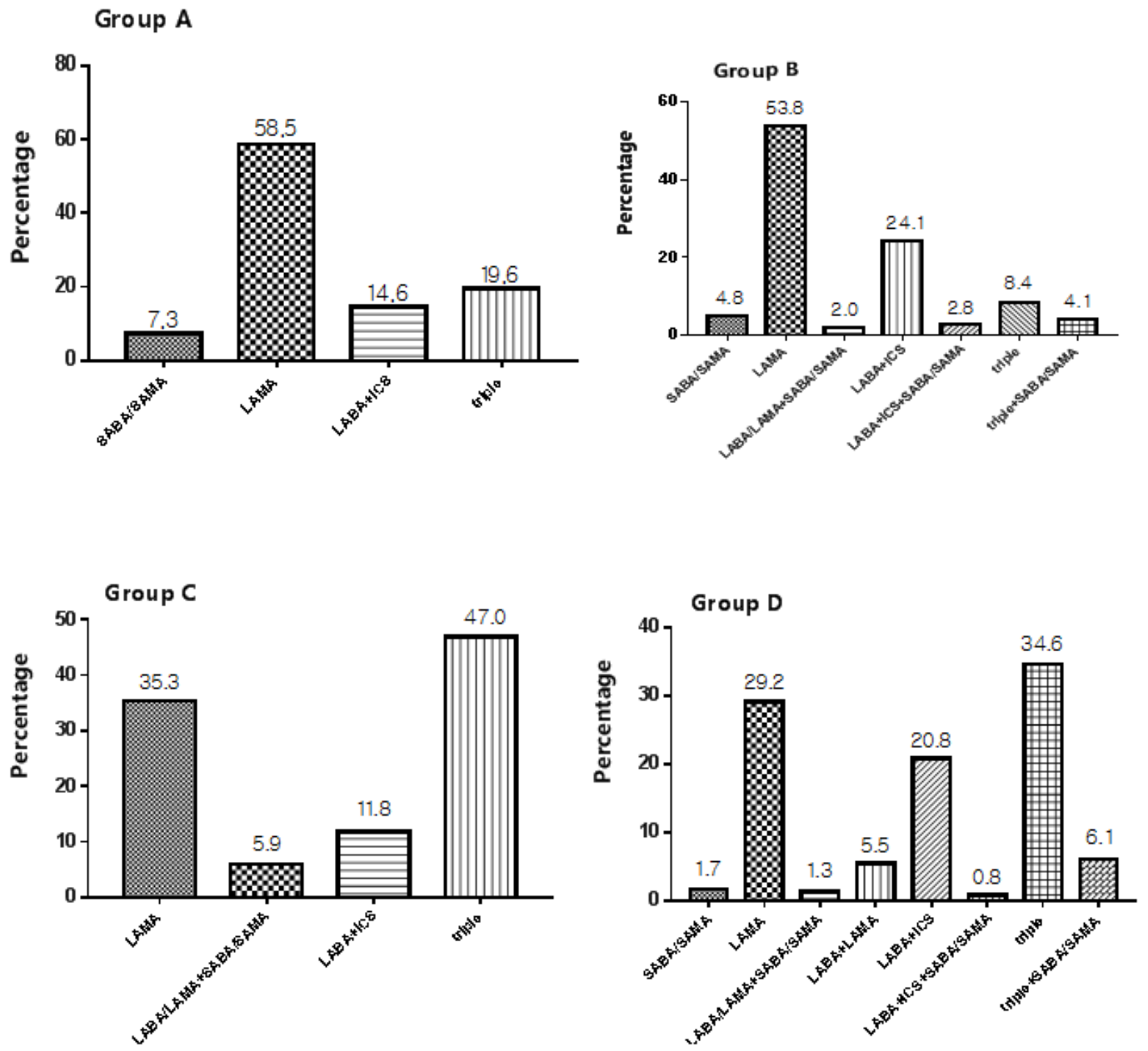

Figure 4

Distribution of medications in Group A, B, C, D 

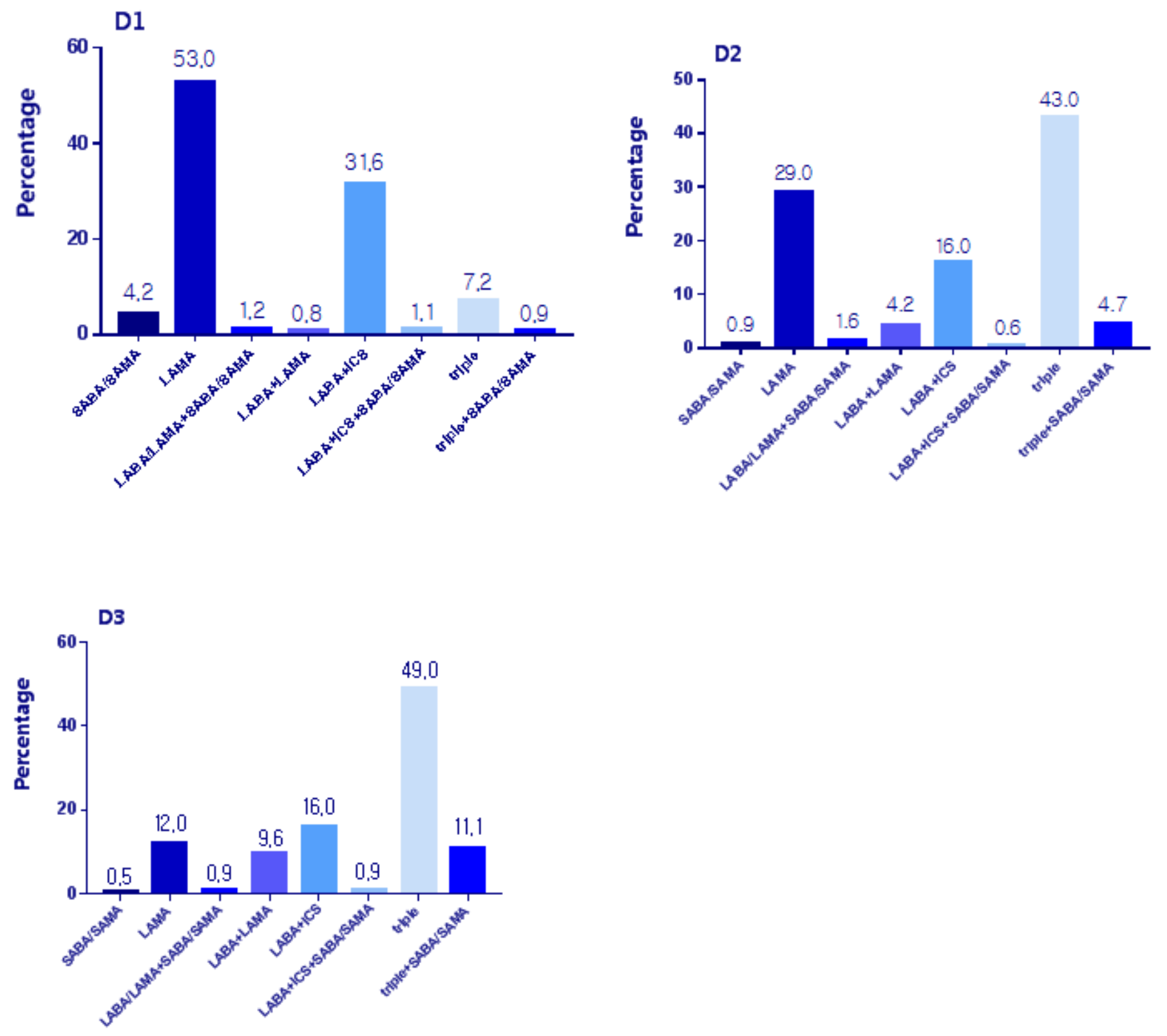

Figure 5

Distribution of medications in Subgroups D1, D2, D3 

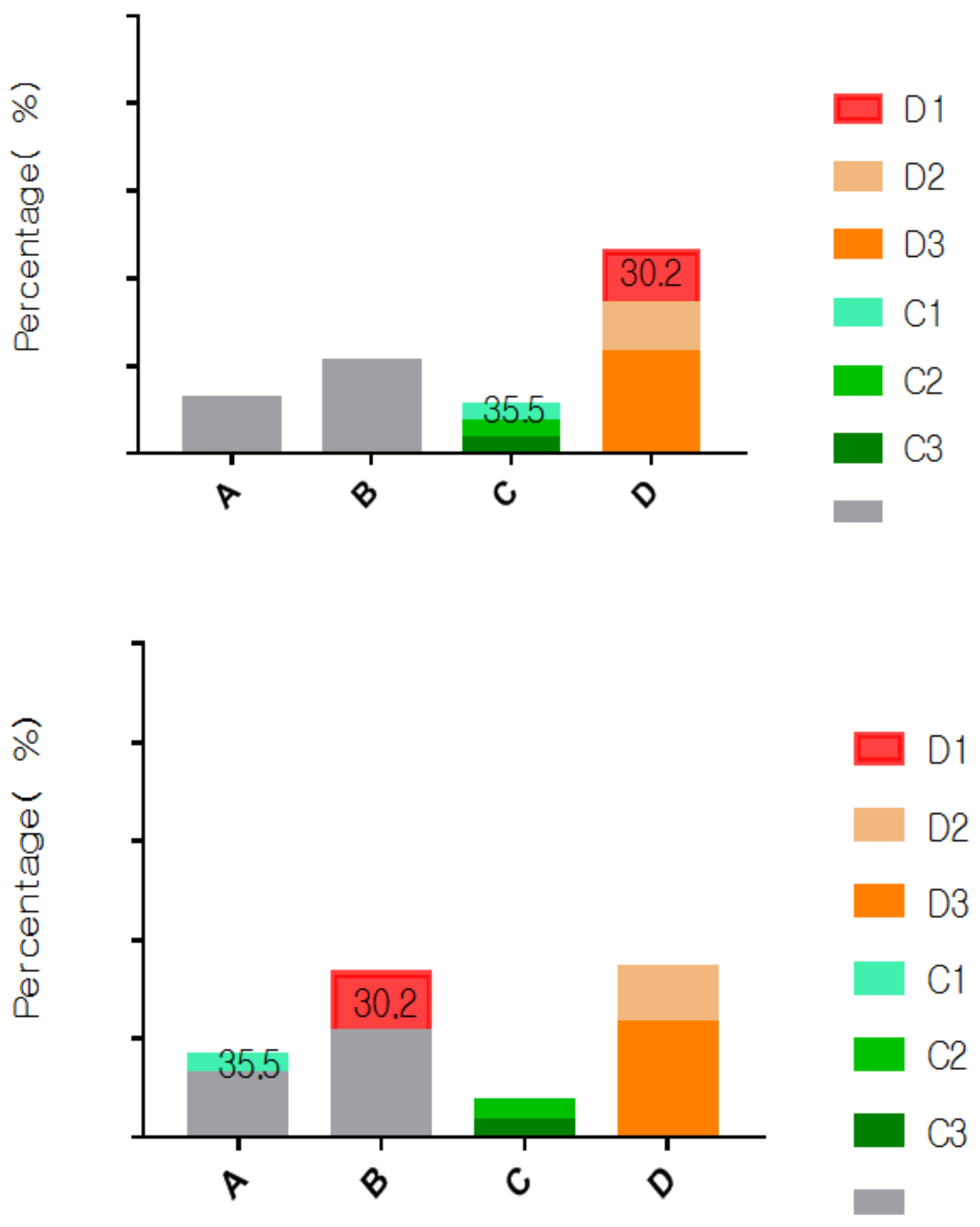

Figure 6

Group transition from GOLD 2016 to GOLD2019 Abbreviation: GOLD, global Initiative for Chronic Obstructive lung Disease. 
$\mathbf{A}$
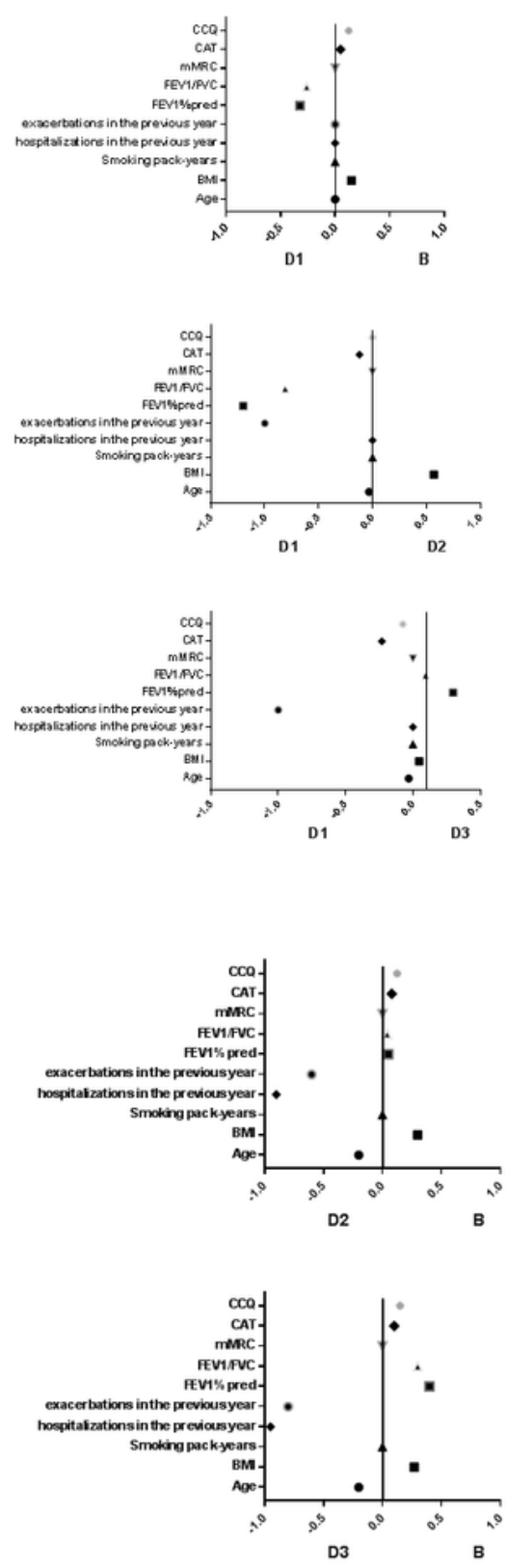
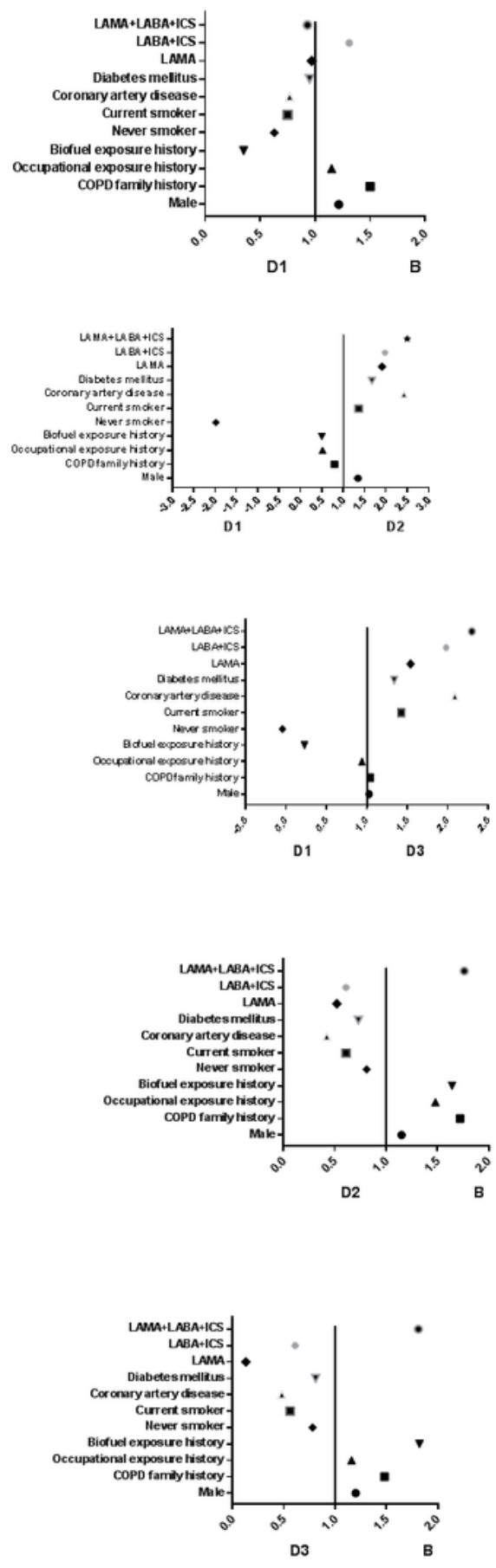

\section{Figure 7}

Difference in demographic, clinical characteristics and medications between subgroup D1, group B, subgroup D2, and subgroup D3. Notes: (A) Forest plot using standardized mean difference of demographic, clinical characteristics and medications for subgroup analysis. (B) Forest plot using odds ratio of demographic, clinical characteristics and medications for subgroup analysis. 


\section{Supplementary Files}

This is a list of supplementary files associated with this preprint. Click to download.

- table1.pdf

- table2.pdf 